\title{
COMPOSITION OPERATORS ON LORENTZ SPACES
}

\author{
S.C. Arora, Gopal Datt and Satish Verma
}

Fredholm, injective, isometric and surjective composition operators on Lorentz spaces $L(p, q)$ are characterised in this paper.

\section{INTRODUCTION}

Let $f$ be a complex-valued measurable function defined on a $\sigma$-finite measure space $(X, \mathcal{A}, \mu)$. For $s \geqslant 0$, define $\mu_{f}$ the distribution function of $f$ as

$$
\mu_{f}(s)=\mu\{x \in X:|f(x)|>s\} \text {. }
$$

By $f^{*}$ we mean the non-increasing rearrangement of $f$ given as

$$
f^{*}(t)=\inf \left\{s>0: \mu_{f}(s) \leqslant t\right\}, t \geqslant 0 .
$$

We also denote the rearrangement of $f$ with respect to the measure $\mu$ by $f^{*, \mu}$. For $t>0$, let

$$
f^{* *}(t)=\frac{1}{t} \int_{0}^{t} f^{*}(s) d s
$$

For $1<p \leqslant \infty, 1 \leqslant q \leqslant \infty$, and for measurable function $f$ on $X$ define $\|f\|_{p q}$ as

$$
\|f\|_{p q}= \begin{cases}\left\{\frac{q}{p} \int_{0}^{\infty}\left(t^{1 / p} f^{* *}(t)\right)^{q} \frac{d t}{t}\right\}^{1 / q}, & 1<p<\infty, 1 \leqslant q<\infty \\ \sup _{t>0} t^{1 / p} f^{* *}(t), & 1<p \leqslant \infty, q=\infty\end{cases}
$$

The Lorentz space denoted by $L(p, q)(X, \mathcal{A}, \mu)$ (or shortly $L(p, q)$ ) is defined to be the vector space of all (equivalence classes of) measurable functions $f$ on $X$ such that $\|f\|_{p q}<\infty$. Also $\|\cdot\|_{p q}$ is a norm and $L(p, q)$ is a Banach space with respect to this norm. The $L^{p}$-spaces for $1<p \leqslant \infty$ are equivalent to the spaces $L(p, p)$. For more on Lorentz spaces one can refer to $[1,2,3,7,10,13,14,15,17]$.

On the measure space $(X, \mathcal{A}, \mu)$, let $T: X \rightarrow X$ be a measurable transformation. Then we define a linear transformation $C_{T}$ on the Lorentz space $L(p, q), 1<p \leqslant \infty$, $1 \leqslant q \leqslant \infty$ into the linear space of all complex-valued measurable functions on $X$ by Received 30th November, 2006

Copyright Clearance Centre, Inc. Serial-fee code: 0004-9727/07 SA2.00+0.00. 
$C_{T} f=f \circ T$. If $C_{T}$ is bounded with range in $L(p, q)$, then it is called a composition operator on $L(p, q)$ induced by $T$. There is a vast literature for composition operators on measurable function spaces and their applications, one can refer to $[4,5,6,8,9,11$, $12,16,18,19,20]$ and references therein.

For a complex-valued measurable function $u$ on $X$, we define a linear transformation $M_{u}$ on the Lorentz space $L(p, q)$ as $M_{u} f=u \cdot f$, where the product of functions is pointwise. If $M_{u}$ is bounded with range in $L(p, q)$, then it is called a multiplication operator on $L(p, q)$ induced by $u$.

For a bounded linear operator $A$ on a Banach space; we use the symbols $N(A)$ and $R(A)$ to denote the kernel and the range of $A$, respectively. We recall that $A$ is called compact if the closure of the image of the unit ball is compact; and Fredholm if $R(A)$ is closed, $\operatorname{dim} N(A)<\infty$ and $\operatorname{codim} R(A)<\infty$, where $\operatorname{dim} N(A)$ is the dimension of $N(A)$ and $\operatorname{codim} R(A)$ is the codimension of $R(A)$, namely the dimension of any subspace complimentary to $R(A)$.

The main aim of this paper is to study Fredholmn property, isometry, invertibility of composition operators on Lorentz spaces $L(p, q)$. In Section 2, we study the boundedness of composition operators between Lorentz spaces with different measure spaces. In Section 3, we discuss the closedness of the range $R\left(C_{T}\right)$, denseness and surjectiveness of composition operator. In Section 4, adjoint of a composition operator is obtained and Fredholm, isometric and invertible composition operators are characterised.

\section{BOUNDEDNESS}

In this section we characterise those measurable transformations $T: Y \rightarrow X$, where $(X, \mathcal{A}, \mu)$ and $(Y, \mathcal{B}, \nu)$ are two $\sigma$-finite measure spaces, for which

$$
C_{T}: L(p, q)(X, \mathcal{A}, \mu) \rightarrow L(p, q)(Y, \mathcal{B}, \nu)(f \mapsto f \circ T)
$$

is bounded.

THEOREM 2.1. A measurable transformation $T: Y \rightarrow X$ induces a composition operator

$$
C_{T}: L(p, q)(X, \mathcal{A}, \mu) \rightarrow L(p, q)(Y, \mathcal{B}, \nu), 1<p \leqslant \infty, 1 \leqslant q \leqslant \infty
$$

if and only if

$$
\left(\nu \circ T^{-1}\right)(E) \leqslant b \mu(E), \text { for all } E \in \mathcal{A}, \text { for some } b>0 .
$$

Moreover

$$
\left\|C_{T}\right\|=k^{1 / p}, \text { where } k=\inf \left\{b_{0}>0:\left(\nu \circ T^{-1}\right)(E) \leqslant b_{0} \mu(E), \text { for all } E \in \mathcal{A}\right\} .
$$


Proof: First assume that $1<p<\infty, 1 \leqslant q<\infty$ and suppose $C_{T}$ is a composition operator induced by $T$. Let $E \in \mathcal{A}, \mu(E)<\infty$. Then the non-increasing rearrangement of the characteristic function $\chi_{E}$ is given by

$$
\chi_{E}^{*}(t)=\chi_{[0, \mu(E))}(t) .
$$

Thus

$$
\begin{aligned}
\chi_{E}^{* *}(t) & =\frac{1}{t} \int_{0}^{t} \chi_{E}^{*}(s) d s \\
& = \begin{cases}1, & \text { if } 0 \leqslant t<\mu(E) \\
\frac{1}{t} \mu(E), & \text { if } t \geqslant \mu(E) .\end{cases}
\end{aligned}
$$

Therefore

$$
\begin{aligned}
\left\|\chi_{E}\right\|_{p q}^{q} & =\frac{q}{p} \int_{0}^{\infty}\left(t^{1 / p} \chi_{E}^{* *}(t)\right)^{q} \frac{d t}{t} \\
& =\mu(E)^{q / p}+\frac{1}{p-1} \mu(E)^{q / p}=p^{\prime}(\mu(E))^{q / p},
\end{aligned}
$$

where $1 / p+1 / p^{\prime}=1$. This implies that $\chi_{E} \in L(p, q)(X, \mathcal{A}, \mu)$ and

$$
\begin{aligned}
\left(\nu \circ T^{-1}\right)(E) & =\nu\left(T^{-1}(E)\right)=\left(p^{\prime}\right)^{-p / q}\left\|\chi_{T^{-1}(E)}\right\|_{p q}^{p} \\
& =\left(p^{\prime}\right)^{-p / q}\left\|\chi_{E} \circ T\right\|_{p q}^{p}=\left(p^{\prime}\right)^{-p / q}\left\|C_{T} \chi_{E}\right\|_{p q}^{p} \\
& \leqslant\left(p^{\prime}\right)^{-p / q}\left\|C_{T}\right\|^{p}\left\|\chi_{E}\right\|_{p q}^{p}=\left\|C_{T}\right\|^{p} \mu(E) .
\end{aligned}
$$

Hence

$$
\left(\nu \circ T^{-1}\right)(E) \leqslant b \mu(E),
$$

where $b=\left\|C_{T}\right\|^{p}$. If $\mu(E)=\infty$, then the inequality is trivial. For $q=\infty, 1<p \leqslant \infty$, we have

$$
\left\|\chi_{E}\right\|_{p \infty}=\sup _{t>0} t^{1 / p} \chi_{E}^{* *}(t)=(\mu(E))^{1 / p} .
$$

Therefore

$$
\left(\nu \circ T^{-1}\right)(E)=\left\|C_{T} \chi_{E}\right\|_{p \infty}^{p} \leqslant\left\|C_{T}\right\|^{p} \mu(E) .
$$

Conversely, suppose there is a constant $b>0$ such that for all $E \in \mathcal{A}$,

$$
\left(\nu \circ T^{-1}\right)(E) \leqslant b \mu(E) .
$$

For $f$ in $L(p, q)(X, \mathcal{A}, \mu)$, the distribution of $f \circ T$ satisfies

$$
\begin{aligned}
\nu_{(f \circ T)}(s) & =\nu\{y \in Y:|f(T(y))|>s\} \\
& =\left(\nu \circ T^{-1}\right)\{x \in X:|f(x)|>s\} \\
& \leqslant b \mu\{x \in X:|f(x)|>s\}=b \mu_{f}(s) .
\end{aligned}
$$


Therefore

$$
\left\{s>0: \mu_{f}(s) \leqslant t\right\} \subseteq\left\{s>0: \nu_{f \circ T}(s) \leqslant b t\right\}
$$

This gives

$$
(f \circ T)^{*, \nu}(b t) \leqslant f^{*, \mu}(t)
$$

and consequently

$$
(f \circ T)^{* *, \nu}(b t) \leqslant f^{* *, \mu}(t), \quad t>0 .
$$

Now for $f$ in $L(p, q), 1<p<\infty, 1 \leqslant q<\infty$,

$$
\begin{aligned}
\left\|C_{T} f\right\|_{p q}^{q} & =\frac{q}{p} \int_{0}^{\infty}\left(t^{1 / p}(f \circ T)^{* *, \nu}(t)\right)^{q} \frac{d t}{t} \\
& =\left(b^{q / p}\right) \frac{q}{p} \int_{0}^{\infty}\left(t^{1 / p}(f \circ T)^{* *, \nu}(b t)\right)^{q} \frac{d t}{t} \\
& \leqslant\left(b^{q / p}\right) \frac{q}{p} \int_{0}^{\infty}\left(t^{1 / p} f^{* *, \mu}(t)\right)^{q} \frac{d t}{t}=\left(b^{q / p}\right)\|f\|_{p q}^{q} .
\end{aligned}
$$

This proves that $C_{T}$ is bounded. For $q=\infty, 1<p \leqslant \infty$, we have

$$
\begin{aligned}
\left\|C_{T} f\right\|_{p \infty} & =\sup _{t>0} t^{1 / p}(f \circ T)^{* *, \nu}(t) \\
& =b^{1 / p} \sup _{t>0} t^{1 / p}(f \circ T)^{* *, \nu}(b t) \\
& \leqslant b^{1 / p} \sup _{t>0} t^{1 / p} f^{* *, \mu}(t)=b^{1 / p}\|f\|_{p \infty} .
\end{aligned}
$$

Hence the result. Moreover, we have

$$
\left\|C_{T}\right\|=k^{1 / p},
$$

where $k=\inf \left\{b_{0}>0:\left(\nu \circ T^{-1}\right)(E) \leqslant b_{0} \mu(E)\right\}$.

COROLlaRY 2.2. ([11]) Let $T: X \rightarrow X$ be a non-singular measurable transformation. Then $T$ induces a composition operator $C_{T}$ on $L(p, q), 1<p<\infty, 1 \leqslant q \leqslant \infty$, if and only if there exists some constant $b>0$ such that

$$
\left(\mu \circ T^{-1}\right)(E) \leqslant b \mu(E), \text { for all } E \in \mathcal{A} .
$$

\section{RANGES OF COMPOSITION OPERATORS}

In this section, we establish conditions for a composition operator to have a closed range or dense range and then we present a characterisation of surjective composition operators.

THEOREM 3.1. If $C_{T}$ is a bounded composition operator on $L(p, q), 1<p \leqslant \infty$, $1 \leqslant q \leqslant \infty$. Then $C_{T}$ has closed range if and only if there exists $\varepsilon>0$ such that $f_{T}(x) \geqslant \varepsilon$ for almost all $x \in S$, where $S=\left\{x \in X: f_{T}(x) \neq 0\right\}$ and $f_{T}$ is the Radon Nikodym derivative of $\mu T^{-1}$ with respect to $\mu$. 
Proof: Suppose $f_{T}(x) \geqslant \varepsilon$ for almost all $x \in S$. Then for $f \in L_{\mu}^{p, q}(S)$, where

$$
\begin{aligned}
L_{\mu}^{p, q}(S) & =\{f \in L(p, q): f \text { vanishes outside } S\}, \\
(f \circ T)^{*}(t) & =\inf \{s>0: \mu\{x \in X:|f(T(x))|>s\} \leqslant t\} \\
& =\inf \left\{s>0: \mu T^{-1}\{x \in S:|f(x)|>s\} \leqslant t\right\} .
\end{aligned}
$$

Now

$$
\mu T^{-1}(E)=\int_{E} f_{T}(x) d \mu \geqslant \varepsilon \mu(E),
$$

where $E=\{x \in S:|f(x)|>s\}$. Hence

$$
\begin{aligned}
(f \circ T)^{*}(\varepsilon t) & \geqslant \inf \{s>0: \mu\{x \in S:|f(x)|>s\} \leqslant t\} \\
& =\inf \{s>0: \mu\{x \in X:|f(x)|>s\} \leqslant t\}=f^{*}(t), \text { for all } t>0
\end{aligned}
$$

and so

$$
(f \circ T)^{* *}(\varepsilon t) \geqslant f^{* *}(t), \text { for all } t>0 .
$$

Therefore

$$
\left\|C_{T}\right\|_{p q} \geqslant \varepsilon^{1 / p}\|f\|_{p q}, \text { for all } f \in L_{\mu}^{p, q}(S) .
$$

As $N\left(C_{T}\right)=L_{\mu}^{p, q}(X \backslash S)$, we get that $C_{T}$ has closed range.

Conversely, suppose that $C_{T}$ has closed range. Then there exists $\varepsilon>0$ such that

$$
\left\|C_{T}\right\|_{p q} \geqslant \varepsilon\|f\|_{p q} \text {, for all } f \in L_{\mu}^{p, q}(S) .
$$

Choose positive integer $n$ such that $1 / n<\varepsilon$. Let $E=\left\{x \in S: f_{T}(x)<1 / n^{p}\right\}$.

If possible $\mu(E)>0$, then $\mu(E)<\left(1 / n^{p}\right) \mu(E)$ and

$$
\left(\chi_{E} \circ T\right)^{*}(t) \leqslant \chi_{E}^{*}\left(n^{p} t\right), \text { for all } t>0 .
$$

This gives

$$
\left\|C_{T} \chi_{E}\right\|_{p q}^{q} \leqslant \frac{1}{n^{q}}\left\|\chi_{E}\right\|_{p q}^{q}<\varepsilon^{q}\left\|\chi_{E}\right\|_{p q}^{q} .
$$

This contradicts (3.1). Hence $f_{T}$ is bounded away from zero.

For a measurable transformation $T$ on measure space $(X, \mathcal{A}, \mu), T^{-1}(\mathcal{A})$ is a $\sigma$-subalgebra of $\mathcal{A}$. Then $L(p, q)\left(X, T^{-1}(\mathcal{A}), \mu\right)$ is a subspace of $L(p, q)$. Now we study the range of composition operators in terms of $L(p, q)\left(X, T^{-1}(\mathcal{A}), \mu\right)$.

THEOREM 3.2. If $C_{T}$ is a composition operator on $L(p, q), 1<p \leqslant \infty$, $1 \leqslant q<\infty$, then the range of $C_{T}$ is dense in $L(p, q)\left(X, T^{-1}(\mathcal{A}), \mu\right)$. 
Proof: In case $X$ is of finite measure, then $\chi_{S}$ in $L(p, q)\left(X, T^{-1}(\mathcal{A}), \mu\right)$ implies that $\chi s=C_{T} \chi_{S^{\prime}}$, for some $S^{\prime} \in \mathcal{A}$. Thus all simple functions of $L(p, q)\left(X, T^{-1}(\mathcal{A}), \mu\right)$ belong to the range of $C_{T}$ and hence using (2.4, Hunt [7]), we find that range of $C_{T}$ is dense in $L(p, q)\left(X, T^{-1}(\mathcal{A}), \mu\right)$. In case $X$ is a $\sigma$-finite measure space, the proof follows from Lebesgue's theorem on dominated convergence.

THEOREM 3.3. A composition operator $C_{T}$ on $L(p, q)$ is surjective if and only if $f_{T}$ is bounded away from zero on its support and $T^{-1}(\mathcal{A})=\mathcal{A}$.

Proof: In case $C_{T}$ is surjective then by using Theorem $3.1, f_{T}$ is bounded away from zero on its support. Let $E \in \mathcal{A}$ be of finite measure. Since $C_{T}$ is surjective, there exists $f \in L(p, q)$ such that $\chi_{E}=C_{T} f$. Then we find $\chi_{E}=\chi_{T^{-1}\left(E_{0}\right) \text {, where }}$ $E_{0}=\{x \in X: f(x)=1\}$. Hence $E=T^{-1}\left(E_{0}\right)$. This proves $\mathcal{A} \subseteq T^{-1}(\mathcal{A})$ and therefore equality. The converse follows by using the Theorems 3.1 and 3.2.

THEOREM 3.4. If $C_{T}$ is a composition operator on $L(p, q), \quad 1<p \leqslant \infty$, $1 \leqslant q<\infty$. Then $C_{T}$ has dense range if and only if $T^{-1}(\mathcal{A})=\mathcal{A}$.

Proof: Suppose $C_{T}$ has dense range. Let $E \in \mathcal{A}$ be such that $\chi_{E} \in L(p, q)$. Then there exists a sequence $\left\langle f_{n}\right\rangle$ in $L(p, q)$ such that $C_{T} f_{n} \rightarrow \chi_{E}$ in $\|\cdot\|_{p q}$ and so $C_{T} f_{n} \rightarrow \chi_{E}$ almost everywhere. Since each $C_{T} f_{n}$ is measurable with respect to $T^{-1}(\mathcal{A})$, therefore $\chi_{E}$ is measurable with respect $T^{-1}(\mathcal{A})$ so that $\chi_{E}=\chi_{T^{-1}(F)}$ for some $F \in \mathcal{A}$. Thus $T^{-1}(\mathcal{A})=\mathcal{A}$.

Conversely suppose $T^{-1}(\mathcal{A})=\mathcal{A}$. Let $E \in \mathcal{A}$ be such that $\mu(E)<\infty$, then we can find $F \in \mathcal{A}$ such that $\mu\left(E \Delta T^{-1}(F)\right)=0$. Since $X$ is $\sigma$-finite, we have an increasing sequence $\left\langle F_{n}\right\rangle$ of measurable set of finite measure such that $F_{n} \uparrow F$ or $T^{-1}\left(F \sim F_{n}\right) \downarrow \phi$. Hence for $\varepsilon>0$, there exists a positive integer $n_{0}$ such that

$$
\mu T^{-1}\left(F \sim F_{n}\right)<\left(\frac{\varepsilon}{p^{\prime}}\right)^{p}, \forall n \geqslant n_{0} .
$$

Hence

$$
\begin{aligned}
\left\|C_{T} \chi_{F}-C_{T} \chi_{F_{n}}\right\|_{p q} & =\left\|C_{T} \chi_{\left(F \sim F_{n}\right)}\right\|_{p q}=\left\|\chi_{T^{-1}\left(F \sim F_{n}\right)}\right\|_{p q} \\
& =p^{\prime}\left(\mu T^{-1}\left(F \sim F_{n}\right)\right)^{1 / p}<\varepsilon, \quad \forall n \geqslant n_{0} .
\end{aligned}
$$

This implies that $\chi_{E} \in \overline{R\left(C_{T}\right)}$. Now the result follows by using [7].

\section{FREDHOLM AND ISOMETRIC COMPOSITION OPERATORS}

In this section we have made an attempt to study the adjoint of the composition operator on $L(p, q), 1<p<\infty, 1 \leqslant q<\infty$. Fredholm, isometric and invertible composition operators are characterised. By using $(2.7$, Hunt $[7$, p. 262]) for every 
$g \in L\left(p^{\prime}, q^{\prime}\right)$, we can find a bounded linear functional $F_{g} \in(L(p, q))^{*}=L\left(p^{\prime}, q^{\prime}\right)$, where $1 / p+1 / p^{\prime}=1=1 / q+1 / q^{\prime}$, defined as

$$
F_{g}(f)=\int f g d \mu, \text { for all } f \in L(p, q) .
$$

For each $g \in L\left(p^{\prime}, q^{\prime}\right)$, there exists a unique $T^{-1}(\mathcal{A})$ measurable function $E(g)$ such that

$$
\int f g d \mu=\int f E(g) d \mu
$$

for each $T^{-1}(\mathcal{A})$ measurable function $f$ for which the left integral exists. $E(g)$ is called the conditional expectation of $g$ with respect to $\sigma$-algebra $T^{-1}(\mathcal{A})$. The Frobenius Perron operator $P_{T}$ on $L\left(p^{\prime}, q^{\prime}\right)$ is defined as

$$
P_{T} g=f_{T} \cdot E(g) \circ T^{-1},
$$

where $E(g) \circ T^{-1}=f$ if and only if $E(g)=f \circ T$.

THEOREM 4.1. If $C_{T}$ is a composition operator on $L(p, q), 1<p \leqslant \infty$, $1 \leqslant q<\infty$, then $C_{T}^{*}$, the adjoint of the composition operator $C_{T}$, is $P_{T}$.

Proof: Let $E \in \mathcal{A}$ be such that $\mu(E)<\infty$. Then for $g \in L\left(p^{\prime}, q^{\prime}\right)$

$$
\begin{aligned}
\left(C_{T}^{*} F_{g}\right)\left(\chi_{E}\right) & =F_{g}\left(C_{T} \chi_{E}\right)=\int C_{T} \chi_{E} \cdot g d \mu \\
& =\int\left(\chi_{E} \circ T\right) \cdot g d \mu=\int E(g) \cdot \chi_{E} \circ T d \mu \\
& =\int E(g) \circ T^{-1} \cdot \chi_{E} d \mu T^{-1}=\int E(g) \circ T^{-1} \cdot \chi_{E} f_{T} d \mu \\
& =F_{\left(E(g) \circ T^{-1}\right) \cdot f_{T}}\left(\chi_{E}\right)
\end{aligned}
$$

Thus $C_{T}^{*} F_{g}=F_{\left(E(g) \circ T^{-1}\right) \cdot f_{T}}$. By identifying $g \in L\left(p^{\prime}, q^{\prime}\right)$ with $F_{g} \in(L(p, q))^{*}$, we can write

$$
C_{T}^{*} g=\left(E(g) \circ T^{-1}\right) \cdot f_{T}=P_{T} g
$$

THEOREM 4.2. If $C_{T}$ is a composition operator on $L(p, q), 1<p \leqslant \infty$, $1 \leqslant q<\infty$, then $N\left(C_{T}^{*}\right)$ is either zero dimensional or infinite dimensional.

Proof: Suppose $0 \neq g \in N\left(C_{T}^{*}\right)$. Let $E=\{x \in X: g(x) \neq 0\}$, then $\mu(E) \neq 0$. Let $\left\langle E_{n}\right\rangle$ be a sequence of disjoint measurable subsets of $E$ such that

$$
E=\bigcup_{n=1}^{\infty} E_{n}, 0<\mu\left(E_{n}\right)<\infty
$$

For each natural number $n$, let $g_{n}=g \cdot \chi_{E} \circ T$. For each $n$,

$$
\begin{aligned}
C_{T}^{*}\left(g_{n}\right) f & =\int\left(g \cdot \chi_{E} \circ T\right)(f \circ T) d \mu \\
& =\int g \cdot\left(\chi_{E} f \circ T\right) d \mu \\
& =C_{T}^{*}(g)\left(\chi_{E} f\right)=0 .
\end{aligned}
$$


Therefore $\left\{g_{n}: n \geqslant 1\right\}$ is a linearly independent subset of $N\left(C_{T}^{*}\right)$. Hence, if $N\left(C_{T}^{*}\right)$ is not zero dimensional, it is infinite dimensional.

Corollary 4.3. If $C_{T}$ is a composition operator on $L(p, q), 1<p \leqslant \infty$, $1 \leqslant q<\infty$. Then $C_{T}$ is injective if and only if $T$ is surjective.

THEOREM 4.4. If $C_{T}$ is a composition operator on $L(p, q), 1<p \leqslant \infty$, $1 \leqslant q<\infty$. Then $C_{T}$ is Fredholm if and only if $C_{T}$ is invertible.

Proof: If $C_{T}$ is Fredholm, then $N\left(C_{T}^{*}\right)$ and $N\left(C_{T}\right)$ both are finite dimensional and are of zero dimension. Therefore $C_{T}$ is injective and $R\left(C_{T}\right)$ is dense in $L(p, q)$. Since $R\left(C_{T}\right)$ is closed, therefore $C_{T}$ is surjective. This proves the invertibility of $C_{T}$. The proof of the converse is obvious.

ThEOREM 4.5. If $C_{T}$ is a composition operator on $L\left(p^{\prime}, q^{\prime}\right)$, then $C_{T}^{*} C_{T}=M_{f_{T}}$.

Proof: On replacing $g$ by $C_{T} g$ in the Theorem 4.1, we find that for every $g \in L\left(p^{\prime}, q^{\prime}\right)$

$$
C_{T}^{*} C_{T} g=C_{T}^{*}(g \circ T)=E(g \circ T) \circ T^{-1} \cdot f_{T}=g \cdot f_{T}=M_{f_{T}} g .
$$

Hence $C_{T}^{*} C_{T}=M_{f_{T}}$.

CoRollary 4.6. If $C_{T}$ is a composition operator on $L(p, q), 1<p \leqslant \infty$, $1 \leqslant q<\infty$, then $C_{T}$ is an isometry if and only if $T$ is measure preserving.

DEFINITION 4.7: ([16]) The essential range of a complex-valued measurable function $f$ defined on the measure space $(X, \mathcal{A}, \mu)$ is given by the set

$$
\{\lambda \in \mathcal{C}: \mu(\{x \in X:|f(x)-\lambda|<\varepsilon\})>0 \text {, for each } \varepsilon>0\} .
$$

By the theory developed so far, we have the following

THEOREM 4.8. If $C_{T}$ is a composition operator on $L(p, q), 1<p \leqslant \infty$, $1 \leqslant q<\infty$, then the following are equivalent:

(1) $C_{T}$ is injective.

(2) $f$ and $f \circ T$ bave the same essential ranges for every $f \in L(p, q)$.

(3) $\mu \ll \mu \circ T^{-1}$.

(4) $f_{T}$ is different from zero almost everywhere.

(5) $M_{f_{T}}$ is injective.

THEOREM 4.9. If $C_{T}$ is a composition operator on $L(p, q), 1<p \leqslant \infty$, $1 \leqslant q<\infty$, then $C_{T}$ is invertible if and only if $f_{T}$ is bounded away from zero almost everywhere on $X$ and $T^{-1}(\mathcal{A})=\mathcal{A}$.

Proof: If $f_{T}$ is bounded away from zero almost everywhere on $X$ and $T^{-1}(\mathcal{A})=\mathcal{A}$, then $M_{f_{T}}$ is injective and hence $C_{T}$ is injective. In view of Theorem 3.3, $C_{T}$ is surjective. Therefore $C_{T}$ is invertible. Converse follows by the Theorems 3.3 and 4.8. 
Invertibility of $T$ does not imply invertibility of $C_{T}$. This is proved by the following examples.

EXAMple 4.10. Let $X=[0,1]$, with the Lebesgue measure $\mu$ on the Borel subsets. Let $T(x)=\sqrt{x}, \forall x \in X$. Then $C_{T}$ is a composition operator on $L(p, q)(111$, Example 5.1]). $U(x)=x^{2}$ is the inverse of T. But $C_{T}$ is not invertible as

$$
\frac{\left\|C_{T} \chi_{[0,1 / n]}\right\|_{p q}^{q}}{\left\|\chi_{[0,1 / n]}\right\|_{p q}^{q}}=\frac{1}{n^{p}},
$$

for each natural number $n$. So $C_{T}$ is not bounded away from zero.

Example 4.11. Let $X=\mathbf{R}$ with Lebesgue measure and let $T(x)=a x+b$, $a \neq 0,1$. Then $T$ is not measure preserving and $C_{T}$ is a composition operator on $L(p, q)$ but $C_{T}$ is not an isometry.

\section{REFERENCES}

[1] R.A. Adams and J.J.F. Fournier, Sobolev spaces, Pure and Applied Math. 140, (Second edition) (Academic Press, New York, 2003).

[2] C. Bennett and R. Sharpley, Interpolation of operators, Pure and Applied Mathematics 129 (Academic Press, London, 1988).

[3] P.L. Butzer and H. Berens, Semigroups of operators and approximation, Die Grundlehren der mathematischen Wissenschaften in Einzeldarstellungen Band 145 (Springer-Verlag, New York, 1967).

[4] J.T. Campbell and J.E. Jamison, "The analysis of composition operators on $L^{p}$ and the Hopf decomposition', J. Math. Anal. Appl. 159 (1991), 520-531.

[5] Y. Cui, H. Hudzik, R. Kumar and L. Maligranda, 'Composition operators in Orlicz spaces', J. Austral. Math. Soc. 76 (2004), 189-206.

[6] T. Hoover, A. Lambert and J. Quinn, 'The Markov process determined by a weighted composition operator', Studia Math. 72 (1982), 225-235.

[7] R.A. Hunt, 'On $L(p, q)$ spaces', L'Enseignment Math. 12 (1966), 249-276.

[8] M.R. Jabbarzadeh and E. Pourreza, 'A note on weighted composition operators on $L^{\text {p }}$ spaces', Bull. Iranian Math. Soc. 29 (2003), 47-54.

[9] B.S. Komal and S. Gupta, 'Composition operators on Orlicz spaces', Indian J. Pure Appl. Math. 32 (2001), 1117-1122.

[10] S.G. Krein, Ju.I. Petunin and E.M. Semenov, Interpolation of linear operators, AMS Translation of Math. Monographs 54 (American Mathematical Society, Providence, RI, 1982).

[11] R. Kumar and R. Kumar, 'Composition operators on Banach function spaces', Proc. Amer. Math. Soc. 33 (2005), 2109-2118.

[12] R. Kumar and R. Kumar, 'Compact composition operators on Lorentz spaces', Math. Vesnik. 57 (2005), 109-112.

[13] J. Lindenstrauss and L. Tzafriri, Classical Banach spaces II, Function Spaces (Springer Verlag, Berlin, New York, 1979). 
[14] G.G. Lorentz, 'Some new function spaces', Ann. of Math. 51 (1950), 37-55.

[15] G.G. Lorentz, 'On the theory of spaces $\Lambda$ ', Pacific J. Math. 1 (1951), 411-429.

[16] R.K. Singh and J.S. Manhas, Composition operators on function spaces, North Holland Math. Studies 179 (North Holland, Amsterdam, 1993).

[17] E.M. Stein and G. Weiss, Introduction to Fourier analysis on Euclidean spaces, Princeton Math. Series 32 (Princeton Univ. Press, Princeton N.J., 1971).

[18] H. Takagi, 'Fredholm weighted composition operators', Integral Equations Operator Theory 16 (1993), 267-276.

[19] H. Takagi and K. Yokouchi, 'Multiplication and composition operators between two $L^{p}$-spaces', in Function Spaces (Edwardsuille, IL 1998), Contemp. Math. 232 (Providence, RI, 1999), pp. 321-338.

[20] X.M. Xu, 'Compact composition operators on $L^{p}(X, \Sigma, \mu)$ ', Adv. in Math. (China) 20 (1991), 221-225.

Department of Mathematics

University of Delhi

Delhi-110007

India

e-mail: scarora@maths.du.ac.in

Department of Mathematics

SGTB Khalsa College

University of Delhi

Delhi-110007

India

e-mail: vermas@maths.du.ac.in

\author{
Department of Mathematics \\ PGDAV College \\ University of Delhi \\ Delhi-110065 \\ India \\ e-mail gopaldatt@maths.du.ac.in
}

